\title{
Correlation of the Genotype of Paragangliomas and Pheochromocytomas with Their Metabolic Phenotype on 3,4-Dihydroxy-6-18 F-Fluoro-L-Phenylalanin PET
}

\author{
H. Christian Rischke*1,2, Matthias R. Benz*1,3 ${ }^{*}$ Damian Wild ${ }^{1}$, Michael Mix ${ }^{1}$, Rebecca A. Dumont ${ }^{1}$, Dean Campbell ${ }^{3}$, \\ Jochen Seufert ${ }^{4}$, Thorsten Wiech ${ }^{5}$, Jochen Rössler ${ }^{6}$, Wolfgang A. Weber ${ }^{1,3}$, and Hartmut P.H. Neumann ${ }^{7}$ \\ ${ }^{1}$ Department of Nuclear Medicine, University of Freiburg, Freiburg, Germany; ${ }^{2}$ Department of Radiation Oncology, University of \\ Freiburg, Freiburg, Germany; ${ }^{3}$ Department of Molecular and Medical Pharmacology, Ahmanson Translational Imaging Division, \\ David Geffen School of Medicine at UCLA, Los Angeles, California; ${ }^{4}$ Department of Medicine, Division of Endocrinology, University \\ of Freiburg, Freiburg, Germany; ${ }^{5}$ Department of Pathology, University of Freiburg, Freiburg, Germany; ${ }^{6}$ Department of Pediatrics, \\ University of Freiburg, Freiburg, Germany; and ${ }^{7}$ Department of Nephrology, Section of Preventive Medicine, University of Freiburg, \\ Freiburg, Germany
}

Paragangliomas and pheochromocytomas are genetically heterogeneous diseases. The purpose of this study was to determine the sensitivity and specificity of PET with 3,4-dihydroxy-6-18 Ffluoro-L-phenylalanin ( $\left.{ }^{18} \mathrm{~F}-\mathrm{DOPA}\right)$ for the detection and staging of pheochromocytomas/paragangliomas. Furthermore, we assessed whether the genotypes of pheochromocytomas and paragangliomas correlate with the uptake of ${ }^{18} \mathrm{~F}-\mathrm{DOPA}$. Methods: We retrospectively analyzed 101 consecutive patients who underwent ${ }^{18} \mathrm{~F}$-DOPA PET or ${ }^{18} \mathrm{~F}$-DOPA PET/CT for known or suspected pheochromocytomas or paragangliomas. Maximum ${ }^{18} \mathrm{~F}$-DOPA tumor uptake was quantified relative to uptake in the liver. Results: Histopathology, cross-sectional imaging, and follow-up indicated the presence of paragangliomas and pheochromocytomas in 68 patients and the absence of a tumor in 33 patients. The average ${ }^{18} \mathrm{~F}$-DOPA uptake by paragangliomas and pheochromocytomas, expressed as a tumor-to-liver ratio, was $5.9 \pm 5.2$. There was no significant difference in uptake among patients with von Hippel Lindau syndrome $(V H L ; n=19)$, succinate dehydrogenase B-D mutation $(n=$ 21), neurofibromatosis type $1(n=1), \operatorname{RET}(n=1)$, no germline mutation $(n=20)$, or unknown mutation status $(n=6)(P=$ 0.84). All 8 patients with an $S D H D$ mutation were true-positive on ${ }^{18} \mathrm{~F}$-DOPA PET. There were 2 cases of false-negative results each in the group with $S D H B(2 / 12)$ and $V H L$ mutations $(2 / 19)$ and 1 false-negative result in the subgroup of patients with unknown mutation status (1/6). Overall, ${ }^{18} \mathrm{~F}-\mathrm{DOPA}$ PET yielded a sensitivity of $93 \%$ and a specificity of $88 \%$ for the detection of paragangliomas and pheochromocytomas on a patient basis (positive and negative predictive value, $94 \%$ and $85 \%$, respectively). Conclusion: ${ }^{18} \mathrm{~F}$-DOPA PET is a sensitive and specific imaging modality for the detection and staging of pheochromocytomas and paragangliomas in different genotypes, including

Received Nov. 27, 2011; revision accepted Apr. 2, 2012

For correspondence or reprints contact: Hartmut P.H. Neumann, Department of Nephrology, Section of Preventive Medicine, University of Freiburg, Hugstetterstrasse 55, 79106 Freiburg, Germany.

E-mail: hartmut.neumann@uniklinik-freiburg.de

${ }^{*}$ Contributed equally to this work.

Published online Jul. 26, 2012.

COPYRIGHT @ 2012 by the Society of Nuclear Medicine and Molecular Imaging, Inc.
$V H L-, S D H B-$, and $S D H D$-mutation carriers, and in patients with no germline mutation.

Key Words: ${ }^{18} \mathrm{~F}-\mathrm{DOPA}$ PET; pheochromocytoma; paraganglioma; VHL; SDHX

J Nucl Med 2012; 53:1352-1358

DOI: 10.2967/jnumed.111.101303

$\mathbf{P}$ heochromocytomas and paragangliomas are tumors arising from sympathetic and parasympathetic paraganglia throughout the body. The World Health Organization defines pheochromocytomas as tumors with an intraadrenal origin, whereas extraadrenal paragangliomas are defined as tumors arising from other locations (1). A more symptomoriented classification defines pheochromocytomas as tumors originating from the sympathetic neuroendocrine system (which can be located in the adrenal medulla or in extraadrenal abdominal, intrapelvic, or thoracic locations), whereas paragangliomas are tumors that derive from the parasympathetic system predominantly located in the head and neck region. These tumors usually do not secrete catecholamines and are also known as glomus tumors (2).

Pheochromocytomas and paragangliomas can occur sporadically or in the context of hereditary syndromes. Approximately $25 \%$ of patients with pheochromocytomas or paragangliomas are carriers of a germline mutation of the following susceptibility genes: RET, von Hippel Lindau $(V H L)$, succinate dehydrogenase B-D $(S D H x)$, or neurofibromatosis type $1(N F 1)(3,4)$. These different mutations are correlated with various biologic properties of pheochromocytomas and paragangliomas: the risk for malignancy ranges from none in $S D H C$, less than $3 \%$ for $S D H D$, and up to about $25 \%$ for $S D H B$ mutation carriers. In addition, the risk for the development of multiple lesions is higher in patients with $V H L$ or $S D H D$ mutation $(55 \%$ 
and $48 \%$, respectively) than in those with $N F 1, S D H B$, or $S D H C$ mutations $(11 \%-12 \%)$ (data taken from the European-American-Pheochromocytoma-ParagangliomaRegistry based in Freiburg).

3,4-dihydroxy-6- ${ }^{18}$ F-fluoro-phenylalanin ( ${ }^{18} \mathrm{~F}$-DOPA) PET has been developed to investigate the activity of the dopaminergic system in neurologic disorders (5). After intracellular uptake, ${ }^{18} \mathrm{~F}$-DOPA is decarboxylated by DOPA decarboxlyase to ${ }^{18} \mathrm{~F}$-dopamine and trapped intracellularly. However, various neuroendocrine tumors also have the ability to accumulate and decarboxylate ${ }^{18} \mathrm{~F}$-DOPA. These tumors include pheochromocytomas, paragangliomas (6), medullary thyroid cancer (7), and insulinomas (8), among others (9). In pheochromocytomas and paragangliomas, ${ }^{18} \mathrm{~F}$-DOPA PET has been used for several years to detect pheochromocytomas, to differentiate pheochromocytomas and paragangliomas from other tumors, and to stage patients with known or suspected malignant pheochromocytomas and paragangliomas. Overall, a high diagnostic accuracy of ${ }^{18}$ F-DOPA PET has been observed in a small series of patients $(6,10)$. However, there are only limited data evaluating whether the uptake of ${ }^{18} \mathrm{~F}$-DOPA and the sensitivity of ${ }^{18} \mathrm{~F}$-DOPA PET correlate with the underlying genotype of pheochromocytomas or paragangliomas $(11,12)$.

Therefore, the aim of this study was to investigate whether the tumor-specific genotype of patients with pheochromocytoma and paraganglioma syndrome affects ${ }^{18} \mathrm{~F}$-DOPA tumor avidity in a large series of patients.

\section{MATERIALS AND METHODS}

We retrospectively analyzed 136 consecutive patients referred between January 2006 and December 2010 who underwent ${ }^{18} \mathrm{~F}$ DOPA PET $(n=108)$ or ${ }^{18} \mathrm{~F}-\mathrm{DOPA}$ PET/CT $(n=28)$ for known or suspected pheochromocytomas or paragangliomas or for surveillance in patients with a prior history of pheochromocytomas or paragangliomas.

The reference standard to determine the presence or absence of tumor lesions was histopathology or cross-sectional imaging (MRI or $\mathrm{CT}$ ), including follow-up imaging. To ensure the correct diagnosis, patient records were reviewed again. Patients with data judged insufficient to establish the diagnosis were removed from the analysis. For this retrospective study, the University of Freiburg Institutional Review Board waived the consent requirements.

\section{Molecular Genetic Analysis}

Genomic DNA was extracted from peripheral blood samples using standard procedures. Genetic analysis of the exonic and flanking intronic regions, including splice sites; mutations for all exons of the VHL (NM_000551.2), SDHB (NM_003000.2), SDHC (NM_003001), and SDHD (NM_003002.2) genes; and exons 10, 11,13 , and 16 of the RET (NM_020975.4) gene was performed as described previously $(4,13)$. In addition, a screening search was performed for large deletions or rearrangements of the genes $V H L$, $S D H B, S D H C$, and SDHD.

\section{${ }^{18} \mathrm{~F}$-DOPA PET and ${ }^{18} \mathrm{~F}-\mathrm{DOPA}$ PET/CT}

At our center, ${ }^{18} \mathrm{~F}$-DOPA PET was introduced for imaging of paragangliomas and pheochromocytomas about $10 \mathrm{y}$ ago (14). Since then, it has been used clinically for their detection and staging. In this study, we retrospectively analyzed these scans and correlated ${ }^{18} \mathrm{~F}$-DOPA uptake with the genotype of the patients.

${ }^{18} \mathrm{~F}-\mathrm{DOPA}$ was synthesized by modifying a previously reported procedure (15). Patients were instructed to fast for at least $6 \mathrm{~h}$ before PET or PET/CT to standardize imaging conditions.

${ }^{18} \mathrm{~F}-\mathrm{DOPA}$ was injected intravenously at an average of $286 \pm$ $55 \mathrm{MBq}$ (median, $296 \mathrm{MBq}$; range, 105-408 MBq). PET was started $47 \pm 18 \mathrm{~min}$ (median, $42 \mathrm{~min}$ ) after radiotracer injection.

Whole-body PET images were acquired in 2-dimensional mode on a dedicated bismuth germinate PET system (2-ring ECAT EXACT 921 or 3-ring ECAT EXACT 922; Siemens/CTI). One-minute transmission scans were obtained with 3 rotating ${ }^{68} \mathrm{Ge}$ line sources for segmented attenuation correction. Eight to 12 bed positions with 5-min emission times each were measured, covering an axial range of $70-100 \mathrm{~cm}(10.8-$ or $16.2-\mathrm{cm}$ axial PET field of view, 1.7-cm bed overlap).

Images were reconstructed with attenuation-weighted orderedsubset expectation maximization ( 2 iterations, 8 subsets, 6-mm gaussian postfiltering representing the default whole-body protocol of the scanner software).

Combined whole-body PET/CT images were acquired on a 64-slice Gemini TF lutetium yttrium orthosilicate PET/CT system (Philips Healthcare). CT acquisition parameters were 120 $\mathrm{kVp}$ and 200-400 mAs (dose-modulation-enabled, depending on patient weight) for contrast-enhanced full diagnostic scans and $25 \mathrm{mAs}$ for low-dose CT. PET images were acquired for 1.5 or 2 min per bed position, depending on patient body weight. CT data were used to correct for photon attenuation.

\section{CT and MRI}

CT was performed on different multislice scanners (Siemens Definition [Siemens Medical Solutions] or Brilliance 64 [Philips]).

MRI was performed on different 1.5-T systems (Magnetom Avanto, Espree, or Symphony; Siemens Medical Solutions), each equipped with a phased-array surface coil (Body Matrix; Siemens Medical Solutions). However, the standard CT protocol consisted of a biphasic contrast-enhanced arterial phase CT scan of the head and neck, thorax, and upper abdomen and a portal-venous phase scan of the abdomen. The slice thickness was $3 \mathrm{~mm}$ in the head and neck and thorax and $4-5 \mathrm{~mm}$ in the abdomen.

The standard MRI protocol comprised an axial T2-weighted spinecho sequence with fat saturation and a native axial T1-weighted spin-echo sequence. Additional coronal T2-weighted spin-echo sequences of the head and neck, and axial and coronal half-Fourier acquisition single-shot turbo spin-echo sequences of the abdomen, were usually part of the sequence protocol. Contrast-enhanced 3-dimensional magnetic resonance angiography was usually performed during the arterial passage of the contrast medium in the head and neck. After contrast injection, coronal and axial T1weighted spin-echo sequences with fat saturation were added for exact localization of the tumor. Because of scanner-specific sequence protocols, the slice thickness of the sequences varied between 3 and $5 \mathrm{~mm}$.

\section{Image Analysis}

All PET images were analyzed by 3 observers. A positive PET scan result was defined as a focal ${ }^{18} \mathrm{~F}$-DOPA accumulation in a location at which physiologic tracer uptake is usually absent. Two observers were aware of the clinical diagnosis and additional imaging studies and performed all quantitative PET ( ${ }^{18} \mathrm{~F}-\mathrm{DOPA}$ tumor uptake) and cross-sectional imaging (tumor size) mea- 
surements. A third observer was unaware of the clinical diagnosis and additional imaging studies and reviewed all PET images with regard to lesion detectability. ${ }^{18} \mathrm{~F}$-DOPA tumor uptake was measured by determination of the maximum tracer uptake in the tumor relative to the average tracer uptake in the liver using ROVER (Region of Interest Visualization, Evaluation, and Image Registration software package; $\mathrm{ABX} \mathrm{GmbH})$. A volume of interest covering the tumor with the highest ${ }^{18} \mathrm{~F}$-DOPA accumulation was defined on PET images. Then, a volume of interest with a diameter of $4 \mathrm{~cm}$ was placed in the liver, excluding the large intrahepatic bile ducts. ${ }^{18} \mathrm{~F}$-FDOPA uptake was expressed as the maximum activity concentration in the lesion divided by the mean activity concentration in the liver. In patients with metastatic or multiple lesions, only the lesion with the highest ${ }^{18} \mathrm{~F}$-DOPA uptake that had histologic or anatomic imaging confirmation (index lesion) was used for further quantitative analyses. All CT and MRI scans were read by 1 board-certified radiologist. Standard diagnostic MRI or CT criteria were applied to correctly classify the tumor lesions.

\section{Statistical Analysis}

Quantitative data are presented as mean $\pm \mathrm{SD}$, median, and range. The Mann-Whitney $U$ test and the Kruskal-Wallis test were used for unpaired comparisons between quantitative parameters. The Spearman rank test was used to correlate 2 linear variables. $P$ values less than 0.05 were considered statistically significant.

Statistical analysis was performed using SPSS software (version 19; IBM).

\section{RESULTS}

\section{Patients and Imaging}

Consecutive patient files $(n=136)$ were reviewed for this study. Thirty-five patients $(26 \%)$ were excluded from the analysis after the data were judged insufficient to establish a definitive diagnosis independent from the findings of the PET scan. The molecular genetic analysis of the excluded patients was as follows: $S D H B, n=4 ; S D H D$, $n=6$; unknown, $n=17$; no mutation, $n=4$; RET, $n=3$; and NF1, $n=1$. In 101 patients, histopathology $(n=37)$ or cross-sectional (MRI, $n=50$; CT, $n=13$ ) or functional (DOTATATE PET follow-up, $n=1$ ) imaging was available as the gold standard to determine the absence $(n=33)$ or presence $(n=68)$ of paraganglioma and pheochromocytoma lesions. Fifty-six patients were men $(55 \%)$ and 45 were women (45\%), with a mean age of $44 \pm 17$ y (median, $45 \mathrm{y}$; range, 7-80 y) (Table 1). The time interval between ${ }^{18} \mathrm{~F}$-DOPA PET or PET/CT and morphologic imaging was $26 \pm 37 \mathrm{~d}$ (median, $12 \mathrm{~d}$; range, 0-180 d).

\section{Correlation Between ${ }^{18} \mathrm{~F}$-DOPA Uptake and Mutation Status}

In 68 patients, histopathology $(n=36)$ or additional imaging (MRI, $n=25$; CT, $n=6$; and DOTATATE follow-up, $n=$ 1) revealed the presence of at least 1 pheochromocytoma or paraganglioma lesion. Genetic testing results for germline mutations were available for 62 of 68 patients (91\%) (Table 2).

To avoid data bias due to systematic variability of image acquisition on different scanners, ratios of maximum tumor uptake to liver were compared in datasets acquired by
TABLE 1

Patient Characteristics $(n=101)$

\begin{tabular}{lc}
\hline Characteristic & $n$ \\
\hline Age (y) & \\
Median & 45 \\
Range & $7-80$ \\
Sex & \\
Male & $56(55)$ \\
Female & $45(45)$ \\
Scanner & \\
ECAT 921 & $7(7)$ \\
ECAT 922 & $72(71)$ \\
PET/CT & $22(22)$ \\
Mutation & $19(19)$ \\
VHL & $20(20)$ \\
SDHB & $2(2)$ \\
SDHC & $11(11)$ \\
SDHD & $1(1)$ \\
NF1 & $1(1)$ \\
RET & $24(24)$ \\
No mutation & $23(23)$ \\
Unknown & \\
Gold standard & $37(37)$ \\
Histology & $50(50)$ \\
MRI & $13(13)$ \\
CT & $1(1)$ \\
DOTATATE PET & \\
&
\end{tabular}

Data in parentheses are percentages.

separate scanners using the Kruskal-Wallis test and were correlated to injected doses and uptake times using the Spearman rank test. The tumor-to-liver ratio did not differ significantly between scanners $(P=0.77)$ and was not correlated to injected dose $(P=0.56)$ or uptake time $(P=$ $0.44)$. Therefore, further analyses were performed by combining the datasets as appropriate.

The tumor-to-liver ratio of all tumors averaged $5.9 \pm 5.2$ (median, 4.2; range, 0-19.2) and did not differ significantly among patients with $V H L$ (mean, $6.9 \pm 5.7$ ), $S D H B$ (mean, $5.9 \pm 5.3$ ), SDHC (0.9), SDHD (mean, $6.0 \pm 7.1$ ), NF1 (2.8), RET (4.9), no germline mutation (mean, $5.8 \pm 4.7$ ), and unknown mutational status (mean, $4.4 \pm 4.1)(P=$ 0.84). A separate comparison of the 4 largest subgroupsVHL $(n=19), \operatorname{SDHB}(n=12), \operatorname{SDHD}(n=8)$, and no mutation $(n=20)$ —confirmed this result $(P=0.81)$ (Fig. 1$)$.

In a patient-based analysis, all 8 patients with $S D H D$ mutation and pheochromocytomas/paragangliomas were classified as true-positive on ${ }^{18} \mathrm{~F}$-DOPA PET. There were 2 cases of false-negative results each in the groups with $S D H B$ and $V H L$ mutations and 1 false-negative metastatic index lesion in the group with unknown mutation status (Table 3). Therefore, 63 of 68 pheochromocytoma/paraganglioma patients were correctly classified by ${ }^{18} \mathrm{~F}$-DOPA PET (sensitivity, 93\%) (Figs. 2 and 3).

Thirty-three patients showed no clinical or morphologic evidence of paragangliomas or pheochromocytomas. However, in 4 of the 33 patients (12\%) PET scans depicted 
TABLE 2

Characteristics of Patients with Proven Pheochromocytomas or Paragangliomas $(n=68)$

\begin{tabular}{|c|c|}
\hline Characteristic & $n$ \\
\hline \multicolumn{2}{|l|}{ Age $(y)$} \\
\hline Median & 42 \\
\hline Range & $7-80$ \\
\hline \multicolumn{2}{|l|}{ Sex } \\
\hline Male & $41(60)$ \\
\hline Female & $27(40)$ \\
\hline \multicolumn{2}{|l|}{ Site of analyzed lesion } \\
\hline Adrenal & $27(40)$ \\
\hline Head and neck & $20(29)$ \\
\hline Other extraadrenal & $21(31)$ \\
\hline \multicolumn{2}{|l|}{ Lesion } \\
\hline Primary & $45(66)$ \\
\hline Multiple & $15(22)$ \\
\hline Metastatic & $8(12)$ \\
\hline \multicolumn{2}{|l|}{ Tumor size $(\mathrm{cm})$} \\
\hline$<3$ & $39(57)$ \\
\hline $3-6$ & $19(28)$ \\
\hline$>6$ & $6(9)$ \\
\hline Not assessed & $4(6)$ \\
\hline \multicolumn{2}{|l|}{ Scanner } \\
\hline ECAT 921 & 4 \\
\hline ECAT 922 & 49 \\
\hline $\mathrm{PET} / \mathrm{CT}$ & 15 \\
\hline \multicolumn{2}{|l|}{ Mutation } \\
\hline$V H L$ & $19(28)$ \\
\hline$S D H B$ & $12(18)$ \\
\hline$S D H C$ & $1(1)$ \\
\hline$S D H D$ & $8(12)$ \\
\hline NF1 & $1(1)^{\prime}$ \\
\hline$R E T$ & $1(1)$ \\
\hline No mutation & $20(29)$ \\
\hline Unknown & $6(9)$ \\
\hline \multicolumn{2}{|l|}{ Gold standard } \\
\hline Histology & $36(53)$ \\
\hline MRI & $25(37)$ \\
\hline $\mathrm{CT}$ & $6(9)$ \\
\hline DOTATATE PET & $1(1)$ \\
\hline
\end{tabular}

Data in parentheses are percentages.

a false-positive ${ }^{18} \mathrm{~F}$-DOPA accumulation. Focal ${ }^{18} \mathrm{~F}$-DOPA accumulation not specific for pheochromocytomas or paragangliomas was verified by cross-sectional imaging and follow-up as an anatomic variant of pancreas morphology $(n=1)$, diverticulum of duodenum $(n=1)$, moderate focal accumulation in paraesophageal tissue without evidence of nodular structure $(n=1)$, or moderate focal accumulation in paracolic tissue without evidence of nodular structure $(n=1)$. Therefore, in a patient-based analysis, the specificity of ${ }^{18} \mathrm{~F}$-DOPA PET was $88 \%$, the positive predictive value was $94 \%$, the negative predictive value was $85 \%$, and the diagnostic accuracy was $91 \%$.

In a lesion-based analysis, ${ }^{18} \mathrm{~F}-\mathrm{DOPA}$ PET detected 180 of the 189 lesions identified by morphologic imaging (95\%). The detection rates for the individual genotypes are summarized in Table 3.

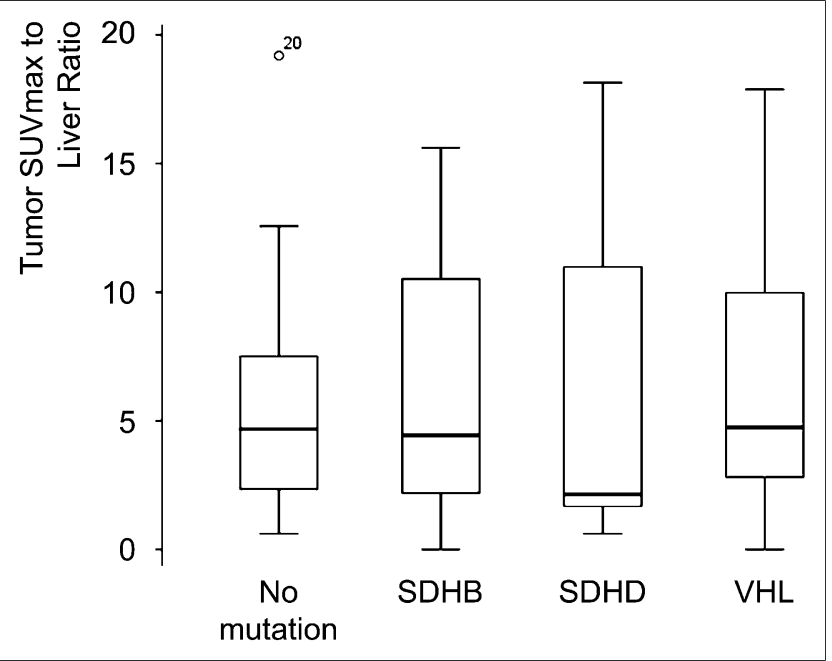

FIGURE 1. Box plots of ratios of tumor maximum standardized uptake value to mean liver showing median, lower and upper quartiles, range, and outlier (patient 20) in patients with and without germline mutations. SUVmax = maximum standardized uptake value.

\section{${ }^{18} \mathrm{~F}$-DOPA Uptake in Correlation to Tumor Size and Tumor Location}

In 64 of the 68 pheochromocytoma/paraganglioma patients, the maximum pheochromocytoma/paraganglioma diameter of the index lesion was assessed via MRI $(n=51)$ or CT $(n=$ 13) and averaged $3.1 \pm 2.6 \mathrm{~cm}$ (median, $2.5 \mathrm{~cm}$; range, $0.8-$ $18.2 \mathrm{~cm}$ ). The maximum tumor diameter differed significantly between genotypes, with larger tumors observed in patients with no germline mutation (mean, $4.2 \pm 3.7 \mathrm{~cm}$; median, 3.3 $\mathrm{cm}$; and range, $1.4-18.2 \mathrm{~cm}$ ) and unknown mutation status (mean, $4.6 \pm 2.4 \mathrm{~cm}$; median, $5.1 \mathrm{~cm}$; and range, $1.2-7.8 \mathrm{~cm}$ ) than in patients with $S D H B$ (mean, $2.9 \pm 1.7 \mathrm{~cm}$; median, 2.5 $\mathrm{cm}$; and range, 1.2-6.5 cm), SDHD (mean, $2.4 \pm 2.6 \mathrm{~cm}$; median, $1.3 \mathrm{~cm}$; and range, $0.9-8.6 \mathrm{~cm}$ ), and VHL mutations (mean, $2.2 \pm 1.2 \mathrm{~cm}$; median, $2.0 \mathrm{~cm}$; and range, $1.1-4.5 \mathrm{~cm}$ ) $(P=0.009)$. The Spearman rank test revealed a weak but significant correlation between maximum tumor diameter and the ${ }^{18}$ F-DOPA tumor-to-liver ratio $(r=0.53 ; P<0.001)$.

TABLE 3

Patient- and Lesion-Based Sensitivity of ${ }^{18} \mathrm{~F}$-DOPA PET According to Underlying Genotype

\begin{tabular}{lcc}
\hline & \multicolumn{2}{c}{ Sensitivity } \\
\cline { 2 - 3 } Genotype & Patient-based & Lesion-based \\
\hline$V H L$ & $17 / 19(89)$ & $24 / 27(89)$ \\
$S D H B$ & $10 / 12(83)$ & $37 / 42(88)$ \\
$S D H C$ & $1 / 1$ & $1 / 1$ \\
$S D H D$ & $8 / 8(100)$ & $29 / 29(100)$ \\
$N F 1$ & $1 / 1$ & $1 / 1$ \\
$R E T$ & $1 / 1$ & $1 / 1$ \\
No mutation & $20 / 20(100)$ & $82 / 82(100)$ \\
Unknown & $5 / 6(83)$ & $5 / 6(83)$
\end{tabular}

Data in parentheses are percentages. 
A

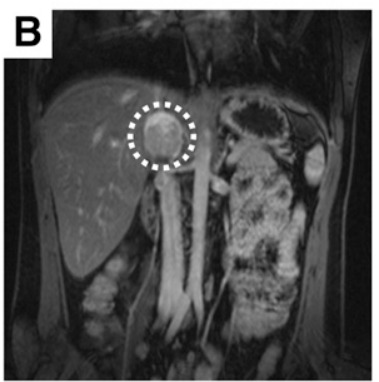

FIGURE 2. (A) True-positive ${ }^{18} \mathrm{~F}$-DOPA PET study of 20 -y-old woman with identified $V H L$ germline mutation. (B) Corresponding MR image of biopsy-proven pheochromocytoma (circled).

Twenty-seven pheochromocytoma/paraganglioma tumor lesions (40\%) were located in the adrenals; 20 in the head or neck (29\%); and $21(31 \%)$ in extraadrenal abdominal, intrapelvic, or thoracic regions. The tumor-to-liver ratio showed no significant correlation with tumor location $(P=0.13)$.

Forty-five patients $(66 \%)$ showed solitary lesions, 15 patients $(22 \%)$ multiple lesions, and $8(12 \%)$ metastatic pheochromocytomas or paragangliomas. In patients with multiple or metastatic disease, only the index lesion was included in the tumor-to-liver ratio analysis.

The sympathetic and parasympathetic nervous systems have a widespread distribution within the human body. Therefore, patients may present with multiple pheochro-
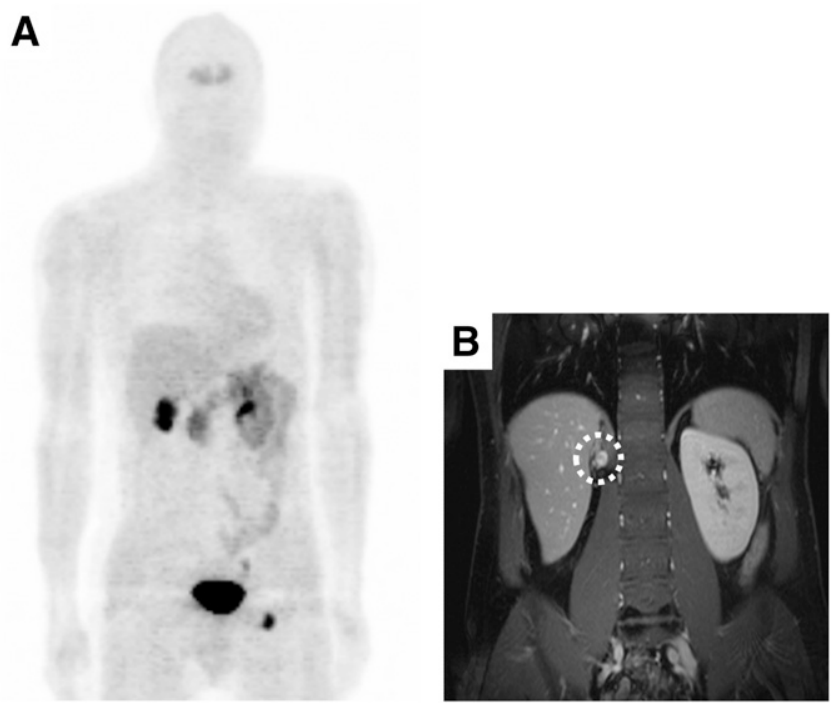

FIGURE 3. (A) False-negative ${ }^{18} \mathrm{~F}$-DOPA PET study of 36 -year-old man with identified $S D H B$ germline mutation (focus in left groin corresponds to urine contamination). (B) MRI revealed presence of biopsy-proven metastatic paraganglioma. Circled area shows presence of biopsy-proven metastatic paraganglioma, which was false-negative in ${ }^{18} \mathrm{~F}$-DOPA PET study. mocytomas/paragangliomas in various areas of the body. The most commonly involved areas include the carotid body, jugular foramen, and middle ear, together with the mediastinum, sympathetic chain, and adrenal medulla. Patients with multiple lesions in these areas were classified as having multifocal disease $(14,16,17)$. Metastatic disease was defined by the presence of lesions outside these areas.

The tumor-to-liver ratio showed no significant difference among patients with solitary lesions (mean, $5.0 \pm 4.5$; median, 3.9; and range, 0-17.9), the index lesion of multiple lesions (mean, $9.3 \pm 6.7$; median, 5.9; and range, 1.519.2), or the index lesion of metastatic lesions (mean, $4.7 \pm$ 3.6; median, 4.1; and range, $0-11.0)(P=0.09)$. The mutational background of multiple and metastatic lesions is provided in Table 4.

\section{DISCUSSION}

In this study, we found no significant influence of germline mutations on tumor ${ }^{18} \mathrm{~F}$-DOPA uptake in a group of 101 consecutive pheochromocytoma/paraganglioma patients. Differences in radiotracer avidity across patients with different germline mutations were small, and there was a much larger variability of ${ }^{18} \mathrm{~F}$-DOPA uptake within each group of mutations. The average tumor-to-liver ratio was not significantly different between solitary lesions and the index lesion of patients with multiple or metastatic pheochromocytomas/ paragangliomas. ${ }^{18} \mathrm{~F}-\mathrm{DOPA}$ uptake was also not significantly influenced by the location of the paraganglioma index lesion. The overall sensitivity and specificity of ${ }^{18} \mathrm{~F}$-DOPA PET on an individual patient basis was $93 \%$ and $88 \%$, respectively. A similarly high sensitivity was observed in a lesion-based analysis $(95 \%)$. These findings indicate that ${ }^{18} \mathrm{~F}-\mathrm{DOPA}$ can be used for the detection and staging of patients with paraganglioma syndrome underlying $V H L, S D H B$, and $S D H D$ mutations and in patients without mutation.

${ }^{18} \mathrm{~F}-\mathrm{DOPA}$ enters cells via the amino acid transporter systems for large neutral amino acids (18). After transmem-

TABLE 4

Genotype of Patients with Multiple or Metastatic Pheochromocytomas or Paragangliomas

\begin{tabular}{lccc}
\hline & \multicolumn{3}{c}{ Patients } \\
\cline { 2 - 4 } Genotype & $n$ & Multiple & Metastatic \\
\hline VHL & 7 & 6 & 1 \\
SDHB & $5^{*}$ & 3 & 4 \\
SDHC & 0 & 0 & 0 \\
SDHD & 4 & 4 & 0 \\
NF1 & 1 & 1 & 0 \\
RET & 0 & 0 & 0 \\
No mutation & 5 & 3 & 2 \\
Unknown & 1 & 0 & 1
\end{tabular}

*Two patients had multiple pheochromocytomas or paragangliomas and metastatic lesions, and therefore numbers do not add up to 5 . 
brane transport, ${ }^{18} \mathrm{~F}$-DOPA is decarboxylated to ${ }^{18} \mathrm{~F}$-fluorodopamine by amino acid decarboxylase, which is strongly expressed in tumors of neuroendocrine or neural origin (19). DOPA uptake is strongly dependent on amino acid decarboxylase and vesicular monoamine transporter 2 expression, as has been shown in preclinical (20) and clinical (21) studies. Several studies have reported the accumulation of ${ }^{18}$ F-DOPA by pheochromocytomas/paragangliomas. Specifically, ${ }^{18} \mathrm{~F}$-DOPA PET is reported to be superior to ${ }^{123} \mathrm{I}-\mathrm{MIBG}$ scintigraphy in the detection of extraadrenal and hereditary pheochromocytomas/paragangliomas (21) and in tumors causing catecholamine excess (22). However, there are currently only limited data on the influence of pheochromocytoma/ paraganglioma genotype on ${ }^{18} \mathrm{~F}$-DOPA uptake. Because the biologic behavior of pheochromocytomas/paragangliomasfor example, risk of malignancy-differs substantially across the different germline mutations, such as risk of malignancy, it is not obvious if the rates of catecholamine synthesis and consequently ${ }^{18} \mathrm{~F}$-DOPA uptake are similar.

In fact, 1 study has found a low sensitivity of ${ }^{18} \mathrm{~F}$-DOPA PET to detect pheochromocytoma/paragangliomas in patients with $S D H B$ mutations. In 15 patients with metastatic pheochromocytomas/paragangliomas, only eight ${ }^{18} \mathrm{~F}-\mathrm{DOPA}$ PET scans were positive (sensitivity on a patient basis, 53\%). In contrast, ${ }^{18}$ F-DOPA PET scans were positive in all 13 patients with metastatic pheochromocytomas/paragangliomas but no $S D H B$ mutation. Similarly, 3 of 4 patients with $S D H B$ mutation and nonmetastatic pheochromocytomas/ paragangliomas had false-negative results on ${ }^{18} \mathrm{~F}$-DOPA PET (patient-based sensitivity, 25\%) (12). In patients with nonmetastatic paraganglioma without $S D H B$ mutations, the sensitivity of ${ }^{18}$ F-DOPA PET was $100 \%$ (16/16 patients). Overall, the sensitivity of ${ }^{18}$ F-DOPA PET to detect pheochromocytomas/paragangliomas was only $47 \%$ (9/19 patients; $95 \%$ confidence interval, $25 \%-71 \%$ ).

In the current study, only 2 of 12 patients with underlying $S D H B$ mutation had false-negative findings (sensitivity, $83 \%$; $95 \%$ confidence interval, 52\%-97\%). One of these patients had a paraganglioma in the paracaval region, and the other in the upper abdominal paraaortal region. The 10 patients with true-positive findings comprised 4 patients with metastatic disease of non-head and neck extraadrenal origin; 4 patients with head and neck lesions, including 1 patient with multiple lesions; 1 patient with a solitary lesion in the adrenal; and 1 patient with a solitary extraadrenal lesion (Table 4).

In accordance with this observation, a recent study has reported that ${ }^{18}$ F-DOPA PET detected 26 of 26 head and neck paragangliomas in 10 patients, including the paragangliomas of 3 patients with $S D H B$ mutation (23). In contrast Timmers et al. reported a low sensitivity of ${ }^{18} \mathrm{~F}$-DOPA PET in patients with $S D H B$ mutations (12). There are several potential explanations for these discrepant findings. First, there are differences with respect to tumor location, because only 1 of $20 S D H B$ mutation carriers in the Timmers study had a lesion located in the head and neck. Second, the following technical factors may explain the differences in reported sensitivities of ${ }^{18} \mathrm{~F}-\mathrm{DOPA}$ PET:

- The studied patient populations with $S D H B$ mutations were relatively small, with wide and overlapping confidence intervals for sensitivity.

- There may have been differences in lesion size that could have influenced the sensitivity of ${ }^{18} \mathrm{~F}$-DOPA PET.

- The available imaging technology may also play a role in lesion detectability. In the present study, all but 1 (false-negative liver metastasis imaged on an integrated PET/CT scanner) false-positive or false-negative ${ }^{18} \mathrm{~F}$ DOPA PET finding was imaged on an older stand-alone PET device.

Third, and perhaps most important, the reference standard used by Timmers et al. (12) included the results of morphologic imaging, ${ }^{18} \mathrm{~F}-\mathrm{FDG}$ PET, ${ }^{123} \mathrm{I}-\mathrm{MIBG}$ SPECT, and ${ }^{18} \mathrm{~F}-$ fluorodopamine PET. This combination of diagnostic tests likely identifies lesions that were missed by routine morphologic imaging studies. Therefore, ${ }^{18} \mathrm{~F}$-DOPA studies in $S D H B$ patients that were considered as true-negative in the present study may have turned out to be false-negative when compared with a reference standard including a combination of multiple functional and morphologic imaging studies.

Larger, preferably multicenter trials are needed to further define the sensitivity of ${ }^{18} \mathrm{~F}$-DOPA in the subgroup of patients with $S D H B$ mutation. However, our study indicates that the variability in ${ }^{18} \mathrm{~F}$-DOPA uptake within a specific genotype is much larger than the variability across groups of patients with a specific germline mutation associated with pheochromocytomas and paragangliomas. This finding suggests that genotype is probably not a major factor influencing ${ }^{18} \mathrm{~F}-\mathrm{DOPA}$ uptake in pheochromocytomas and paragangliomas.

This study has several limitations. First, as with all retrospective analyses, selection bias is a potential concern regarding the interpretation of our findings. Hypothetically, ${ }^{18} \mathrm{~F}-\mathrm{FDG}-$ or ${ }^{123} \mathrm{I}-\mathrm{MIBG}$-positive patients could have been less likely to undergo ${ }^{18} \mathrm{~F}$-DOPA PET, potentially biasing our findings in favor of ${ }^{18} \mathrm{~F}$-DOPA PET. However, ${ }^{18} \mathrm{~F}$-DOPA has been the standard radiotracer in our clinic to study pheochromocytomas and paragangliomas for several years. ${ }^{123}$ I-MIBG SPECT or ${ }^{18}$ F-FDG PET has only rarely performed in this patient population. Our results are therefore unlikely to be prone to a selection bias.

Second, PET scans were acquired on 3 generations of PET scanners with varying uptake times and injected doses. This is because pheochromocytoma/paraganglioma is a relatively rare disease, and it thus took several years to recruit the large series of patients described in this study. However, the ${ }^{18}$ F-DOPA tumor-to-liver ratio did not correlate with injected dose $(P=0.56)$ or uptake time $(P=0.44)$, nor did it correlate with the PET devices $(P=0.77)$. Nevertheless, these confounding factors may have contributed to the variability of ${ }^{18} \mathrm{~F}$-DOPA uptake observed in the present study. These factors may have masked small systematic 
differences in radiotracer uptake across different groups of patients. However, it is unlikely that such small differences in radiotracer uptake will significantly affect lesion detectability in clinical studies.

Third, histopathologic confirmation of a pheochromocytoma or paraganglioma was available in only 36 of 68 patients because of the retrospective nature of this study and the fact that biopsies were not clinically indicated in the remaining patients. However, standard diagnostic criteria for MR or CT images and all available clinical information were used to come to a definitive diagnosis. The University Hospital Freiburg is a center for patients with pheochromocytoma/paraganglioma syndromes and has extensive patient records that were reviewed to ensure the correct diagnosis. Because patients with $S D H x$ or $V H L$ mutations are at high risk for developing pheochromocytomas/paragangliomas, typical clinical and imaging findings on MRI and CT make pheochromocytomas/paragangliomas likely. Accordingly, the rate of false-positive diagnoses can be expected to be low.

Fourth, a recent study in pheochromocytomas/paragangliomas reported (24) increased detection rates due to enhanced ${ }^{18} \mathrm{~F}-\mathrm{DOPA}$ tumor uptake and significantly decreased tracer uptake by the pancreas (higher tumor-to-background ratio) when carbidopa is administered before the ${ }^{18} \mathrm{~F}$-DOPA injection. In the current study, we did not administer DOPA decarboxylase inhibitors, possibly influencing qualitative and quantitative PET measurements.

However, we believe that with the development of efficient and automated nucleophilic syntheses for ${ }^{18} \mathrm{~F}-\mathrm{DOPA}$, the clinical availability of ${ }^{18} \mathrm{~F}$-DOPA PET is expected to increase significantly (25). Therefore, ${ }^{18}$ F-DOPA PET/CT may become the preferred imaging modality for the detection and staging of paragangliomas and pheochromocytomas.

\section{CONCLUSION}

${ }^{18} \mathrm{~F}-\mathrm{DOPA}$ PET is a sensitive and specific imaging modality for the detection and staging of pheochromocytomas/paragangliomas in various genotypes, including $V H L-, S D H B$-, and $S D H D$-mutation carriers, and in patients with no germline mutation.

\section{DISCLOSURE STATEMENT}

The costs of publication of this article were defrayed in part by the payment of page charges. Therefore, and solely to indicate this fact, this article is hereby marked "advertisement" in accordance with 18 USC section 1734.

\section{ACKNOWLEDGMENT}

No potential conflict of interest relevant to this article was reported.

\section{REFERENCES}

1. DeLellis R, Lloyd R, Heitz P. Pathology and Genetics: Tumours of Endocrine Organs (IARC WHO Classification of Tumours). Lyon, France: IARC Press; 2004.

2. Reisch N, Peczkowska M, Januszewicz A, Neumann HP. Pheochromocytoma: presentation, diagnosis and treatment. J Hypertens. 2006;24:2331-2339.
3. Amar L, Bertherat J, Baudin E, et al. Genetic testing in pheochromocytoma or functional paraganglioma. J Clin Oncol. 2005;23:8812-8818.

4. Neumann HP, Bausch B, McWhinney SR, et al. Germ-line mutations in nonsyndromic pheochromocytoma. N Engl J Med. 2002;346:1459-1466.

5. Calne DB, Langston JW, Martin WR, et al. Positron emission tomography after MPTP: observations relating to the cause of Parkinson's disease. Nature. 1985;317:246-248.

6. Hoegerle S, Nitzsche E, Altehoefer C, et al. Pheochromocytomas: detection with ${ }^{18}$ F DOPA whole body PET-initial results. Radiology. 2002;222:507-512.

7. Hoegerle S, Altehoefer C, Ghanem N, Brink I, Moser E, Nitzsche E. ${ }^{18}$ F-DOPA positron emission tomography for tumour detection in patients with medullary thyroid carcinoma and elevated calcitonin levels. Eur J Nucl Med. 2001;28:64-71.

8. Kauhanen S, Seppanen M, Minn H, et al. Fluorine-18-L-dihydroxyphenylalanine $\left({ }^{18} \mathrm{~F}\right.$-DOPA) positron emission tomography as a tool to localize an insulinoma or beta-cell hyperplasia in adult patients. J Clin Endocrinol Metab. 2007;92: 1237-1244.

9. Jager PL, Chirakal R, Marriott CJ, Brouwers AH, Koopmans KP, Gulenchyn KY. 6-L- ${ }^{18} \mathrm{~F}$-fluorodihydroxyphenylalanine PET in neuroendocrine tumors: basic aspects and emerging clinical applications. J Nucl Med. 2008;49:573-586.

10. Imani F, Agopian VG, Auerbach MS, et al. ${ }^{18} \mathrm{~F}-\mathrm{FDOPA}$ PET and PET/CT accurately localize pheochromocytomas. J Nucl Med. 2009;50:513-519.

11. Timmers HJ, Kozupa A, Chen CC, et al. Superiority of fluorodeoxyglucose positron emission tomography to other functional imaging techniques in the evaluation of metastatic SDHB-associated pheochromocytoma and paraganglioma. J Clin Oncol. 2007;25:2262-2269.

12. Timmers HJ, Chen CC, Carrasquillo JA, et al. Comparison of ${ }^{18} \mathrm{~F}$-fluoro-LDOPA, ${ }^{18}$ F-fluoro-deoxyglucose, and ${ }^{18} \mathrm{~F}$-fluorodopamine PET and ${ }^{123} \mathrm{I}-\mathrm{MIBG}$ scintigraphy in the localization of pheochromocytoma and paraganglioma. J Clin Endocrinol Metab. 2009;94:4757-4767.

13. Schiavi F, Boedeker CC, Bausch B, et al. Predictors and prevalence of paraganglioma syndrome associated with mutations of the SDHC gene. JAMA. 2005;294:2057-2063.

14. Hoegerle S, Ghanem N, Altehoefer C, et al. ${ }^{18} \mathrm{~F}-\mathrm{DOPA}$ positron emission tomography for the detection of glomus tumours. Eur J Nucl Med Mol Imaging. 2003;30:689-694.

15. Luxen A, Perlmutter M, Bida GT, et al. Remote, semiautomated production of 6- $\left[{ }^{18} \mathrm{~F}\right]$ fluoro-L-dopa for human studies with PET. Int J Rad Appl Instrum [A]. 1990;41:275-281.

16. Grufferman S, Gillman MW, Pasternak LR, Peterson CL, Young WG Jr. Familial carotid body tumors: case report and epidemiologic review. Cancer. 1980;46: 2116-2122.

17. Tischler AS, Dichter MA, Biales B, Greene LA. Neuroendocrine neoplasms and their cells of origin. N Engl J Med. 1977;296:919-925.

18. Verrey F. System L: heteromeric exchangers of large, neutral amino acids involved in directional transport. Pflugers Arch. 2003;445:529-533.

19. Gazdar AF, Helman LJ, Israel MA, et al. Expression of neuroendocrine cell markers L-dopa decarboxylase, chromogranin A, and dense core granules in human tumors of endocrine and nonendocrine origin. Cancer Res. 1988;48: 4078-4082.

20. Lee WY, Chang JW, Nemeth NL, Kang UJ. Vesicular monoamine transporter-2 and aromatic L-amino acid decarboxylase enhance dopamine delivery after L-3, 4-dihydroxyphenylalanine administration in Parkinsonian rats. J Neurosci. 1999; 19:3266-3274.

21. Fottner C, Helisch A, Anlauf M, et al. 6- ${ }^{18}$ F-fluoro-L-dihydroxyphenylalanine positron emission tomography is superior to ${ }^{123}$ I-metaiodobenzyl-guanidine scintigraphy in the detection of extraadrenal and hereditary pheochromocytomas and paragangliomas: correlation with vesicular monoamine transporter expression. J Clin Endocrinol Metab. 2010;95:2800-2810.

22. Fiebrich HB, Brouwers AH, Kerstens MN, et al. 6-[F-18]Fluoro-L-dihydroxyphenylalanine positron emission tomography is superior to conventional imaging with ${ }^{123}$ I-metaiodobenzylguanidine scintigraphy, computer tomography, and magnetic resonance imaging in localizing tumors causing catecholamine excess. J Clin Endocrinol Metab. 2009;94:3922-3930.

23. King KS, Chen CC, Alexopoulos DK, et al. Functional imaging of SDHx-related head and neck paragangliomas: comparison of ${ }^{18} \mathrm{~F}$-fluorodihydroxyphenylalanine, ${ }^{18} \mathrm{~F}$-fluorodopamine, ${ }^{18} \mathrm{~F}$-fluoro-2-deoxy-D-glucose PET, ${ }^{123}$ I-metaiodobenzylguanidine scintigraphy, and ${ }^{111}$ In-pentetreotide scintigraphy. J Clin Endocrinol Metab. 2011;96:2779-2785.

24. Timmers HJ, Hadi M, Carrasquillo JA, et al. The effects of carbidopa on uptake of 6- ${ }^{18}$ F-Fluoro-L-DOPA in PET of pheochromocytoma and extraadrenal abdominal paraganglioma. J Nucl Med. 2007;48:1599-1606.

25. Shen B, Ehrlichmann W, Uebele M, Machulla HJ, Reischl G. Automated synthesis of n.c.a. $\left[{ }^{18} \mathrm{~F}\right]$ FDOPA via nucleophilic aromatic substitution with $\left[{ }^{18} \mathrm{~F}\right]$ fluoride. Appl Radiat Isot. 2009;67:1650-1653. 